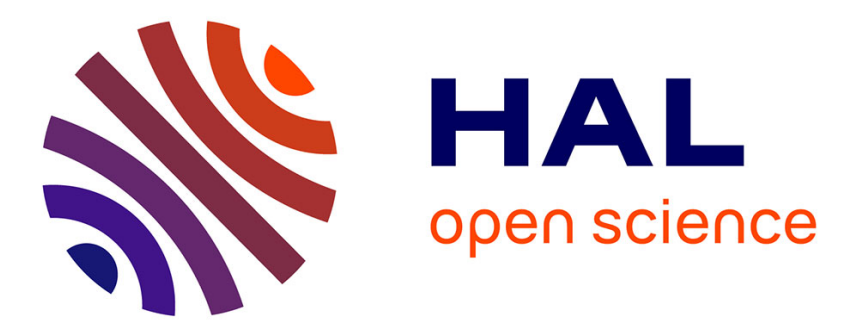

\title{
A Mathematical Model for HVLV Systems Scheduling and Optimization With Periodic Preventive Maintenance Using $(\max ,+)$ Algebra
}

Imed Nasri, Reda Boukezzoula, Georges Habchi

\section{- To cite this version:}

Imed Nasri, Reda Boukezzoula, Georges Habchi. A Mathematical Model for HVLV Systems Scheduling and Optimization With Periodic Preventive Maintenance Using $(\max ,+)$ Algebra. 14th IFAC Symposium on Information Control Problems in Manufacturing (INCOM 2012), May 2012, Bucharest, Romania. pp. 1035-1040. hal-00707441

\section{HAL Id: hal-00707441 \\ https://hal.science/hal-00707441}

Submitted on 8 Jan 2013

HAL is a multi-disciplinary open access archive for the deposit and dissemination of scientific research documents, whether they are published or not. The documents may come from teaching and research institutions in France or abroad, or from public or private research centers.
L'archive ouverte pluridisciplinaire HAL, est destinée au dépôt et à la diffusion de documents scientifiques de niveau recherche, publiés ou non, émanant des établissements d'enseignement et de recherche français ou étrangers, des laboratoires publics ou privés. 


\title{
A Mathematical Model for HVLV Systems Scheduling and Optimization With Periodic Preventive Maintenance Using $(\max ,+)$ Algebra
}

\author{
Imed NASRI* Reda BOUKEZZOULA ** \\ Georges HABCHI *** \\ * University of Savoie, Domaine Universitaire, BP 80439, 74944 \\ Annecy le Vieux, France (e-mail: imed.nasri@univ-savoie.fr). \\ ** University of Savoie, Domaine Universitaire, BP 80439, 74944 \\ Annecy le Vieux, France (e-mail: reda.boukezzoula@univ-savoie.fr) \\ *** University of Savoie, Domaine Universitaire, BP 80439, 74944 \\ Annecy le Vieux, France (e-mail: georges.habchi@univ-savoie.fr)
}

\begin{abstract}
The High-Variety, Low-Volume (HVLV) scheduling problem is one of the most arduous combinatorial optimization problems. This paper considers an interesting formulation of the HVLV scheduling problem using $(\max ,+)$ algebra while periodic Preventive Maintenance $(\mathrm{PM})$ is considered. Maintenance is time based since activities are periodically fixed: maintenance is required after a periodic time interval (all periods are equals on each machine). In this paper, the maintenance tasks of machines are controllable. The jobs and the maintenance operations are scheduled simultaneously. Also, the maintenance operations are scheduled between each other, so that a regular criterion is optimized. To generate feasible schedules, constrained decision variables are incorporated into the $(\max ,+)$ model. The validity of the proposed approach is illustrated by simulation examples.
\end{abstract}

Keywords: HVLV manufacturing systems, (max, +) model, scheduling and control modeling, decision variables, non-linear optimization, periodic preventive maintenance tasks, makespan.

\section{INTRODUCTION}

High-Variety, Low-Volume (HVLV) manufacturing systems are a class of dynamic systems where the behaviour can be assimilated to Discrete Event Dynamic Systems (DEDS). They are characterized by a wide variety of products using shared machines, a weak and personalized demand, relatively long processing times and frequent change over and set-up times. Consequently, a continuous approximation of the production flow by continuous flow systems (Tamani et al. (2009)), (Tamani et al. (2011b)) and (Tamani et al. (2011a)) is not appropriate for HVLV systems. In this framework, it seems very interesting to handle this kind of systems as Job-Shop systems (Huang and Irani (2003)) due to the wide variety of processed products.

DEDS Decision-free according to synchronization problems, where scheduling problem is not considered, has been largely investigated in dioid algebra literature (Houssin et al. (2007)), (Nasri et al. (2011a)) and (Nasri et al. $(2011 b))$. In this framework, the different proposed models are linear.

Generally, production scheduling problems are done to allocate a limited set of resources to a limited number of jobs optimizing the system performances according to one or more criteria where various constraints are taken into consideration (Kusiak and Ahn (1992)). In order to deal with sequencing decisions, control variables have been introduced in the model (Nasri et al. (2011c)). In this context, the dioid algebraic model has been developed to generate all feasible schedules by choosing different values for decision variables. This model is non-linear in the sense of $(\max ,+)$ algebra.

Most of researches assume that machines are always available. A more realistic scheduling model should consider machines availability constraints which improve the frequency of rescheduling. Maintenance activities, especially Preventive Maintenance (PM), can restore the reliability of machines before they go to failure. However, industrial applications cannot ignore forecasted maintenance operations on the machines. Therefore, the problem of simultaneously scheduling production jobs and maintenance activities have been taken great attentions in recent years where the starting times and durations of maintenance tasks are fixed and known in advance (Zribi et al. (2008)) and (Sbihi and Varnier (2008)).

Our contribution in this paper consists in an analytical formulation of a dynamic scheduling of Job-Shop HVLV systems with maintenance tasks using the $(\max ,+)$ algebra. Moreover, Preventive Maintenance (PM) is integrated into the proposed model using simple mathematical relations in the $(\max ,+)$ algebra. Compared to (Sbihi and Varnier (2008)) where the authors consider equal durations of maintenance tasks in the case of single machine scheduling, 
in this paper the allocated times to maintenance operations can be different on each machine. Indeed, the (max, $+)$ model is a simple representation where only sequencing type decisions are needed to solve the conflicts between concurrent operations. In this framework, sequencing operations and maintenance activities are determined by incorporating decision variables in the model. In addition, different kinds of maintenance operations are scheduled via control variables, so that the makespan is minimized.

The remainder of this paper is organized as follows. Section 2 provides a short reminder for HVLV systems scheduling modeling. In Section 3, Preventive Maintenance (PM) is considered in the proposed model. In Section 4, an illustrative example of a $(6 x 6)$ Job-Shop HVLV system with PM according to a non-linear optimization procedure is presented. Concluding remarks and future research directions are presented in Section 5.

\section{HVLV SYSTEMS STATIC SCHEDULING MODELING}

The focus of this section concerns a review for the HVLV systems scheduling using (max, + ) algebra. For more details, readers are invited to read (Nasri et al. (2011c)).

\subsection{Approach Principle}

The approach presented in (Nasri et al. (2011c)) is a direct systematic procedure, relevant to a wide class of manufacturing systems, especially HVLV systems. The technique used to solve the scheduling problem of HVLV systems considers the problem as a mathematical programming formulation while using decision variables. These control variables introduce a non-linearity in the proposed model due to their multiplication by the state variables (starting times of operations). They are used to solve the conflicts between concurrent operations to be processed on the same machines.

$(\mathrm{Max},+)$ algebra is applied as a modeling tool in order to represent the scheduling problem of HVLV systems where relationships between the starting times of the operations require the maximum and addition operators. In order to generate feasible schedules on machines, the control variables used in the proposed model in the case of minimization of the makespan are the decision variables.

A dioid is considered as a set D with two operators, $\oplus$ and $\otimes$. The operation $\oplus$ called addition, produces in $\mathrm{D}$ a structure of a commutative monoid and has a neutral element $\epsilon$ called zero. The other operation, $\otimes$, called multiplication, produces in D a structure of a monoid and has a neutral element $e$, called unity. (Max, + ) is a dioid, which consists of the real numbers $R$ extended to include $-\infty$. (Max, +) algebra is used in development of algebraic models of DEDS (Baccelli et al. (1992)). For all $a, b \in R \cup$ $-\infty$ the max-plus operators are defined according to the following equations:

$$
\begin{gathered}
a \oplus b=\max (a, b) \\
a \otimes b=a+b
\end{gathered}
$$

\subsection{Max-Plus Scheduling Model for HVLV Systems}

Let us now firstly present the construction principle of the $(\max ,+)$ algebraic model for the static (without maintenance) scheduling problem for the HVLV systems. The knowledge of the following informations are needed to establish our model:

- The individual operations for each job.

- The machines on which each operation should be executed.

- The predecessors of each operation (the process plan for each job).

- External starting conditions of each operation (the times at which raw materials are fed to the system and the starting date of a new scheduling in a new planning horizon).

Compared to (Dolgui et al. (2012)) where the authors consider single machine scheduling problems of minimizing the makespan in which the processing time of a job depends on its position, in this paper we consider a deterministic scheduling of HVLV systems where processing times of products are fixed in advance. In this situation, the used model contains three parts: the first one considers initial conditions (exogenous conditions of the beginning of the scheduling), the second one describes precedence requirements of the operations and the third one incorporates decision variables which generate all feasible sequencing on the same machines in our system.

The incorporation of the decision variables into the model is satisfied by the fact that the sequencing of operations for different products on the same machine requires a decision on the order in which the operations are processed such that the conflicts are resolved and precedence constraints are not violated. Moreover, to get feasible schedules, constraints are added in order to bound decision variables.

It was shown in (Nasri et al. (2011c)) that the developed event-timing-equations describing the dynamic of the system can be grouped into the following (max, +) matrix form:

$$
X=T \oplus U \oplus C \otimes X
$$

where:

- $X$ is a $(N \mathrm{x} 1)(\max ,+)$ state vector $(N$ is the total number of operations) which collects the starting times of operations.

- $T$ is a $(N \mathrm{x} 1)(\max ,+)$ vector which is composed of the beginning dates of the scheduling over the new planning horizon.

- $U$ is a $(N \times 1)$ vector which contains the different dates at which the raw material of each product is fed to the system.

- $C$ is a $(N \times N)$ appropriate $(\max ,+)$ matrix describing the relationships among different state variables of the system. It contains the different decision variables. 


\section{STATEMENT OF HVLV SYSTEMS SCHEDULING} PROBLEM WITH MAINTENANCE USING $($ MAX, + ) ALGEBRA

The HVLV system scheduling problem with maintenance activities that we addressed here can be described as follows:

A set of $n$ jobs $J=\left\{J_{1}, J_{2}, \ldots, J_{n}\right\}$ is to be processed on a set of $m$ machines denoted by $M=\left\{M_{1}, M_{2}, \ldots, M_{m}\right\}$. Each job $i$ consists of a sequence of $n_{j}$ operations (routing). Each operation $O_{i j k}\left(1 \leqslant i \leqslant n, 1 \leqslant j \leqslant n_{j}, 1 \leqslant k \leqslant m\right)$ has to be performed to complete one job. There are $h(h=1, \ldots, x)$ periodic maintenance activities that have to be processed on each machine $M_{k}$ during the planning horizon based on a certain maintenance policy. Maintenance periods are periodically fixed: maintenance is required after a periodic time interval (e.g., periodical maintenance with $m$ equal periods $T_{k}(k=1, \ldots, m)$ on each machine $\left.M_{k}\right)$ (see Figure 1).

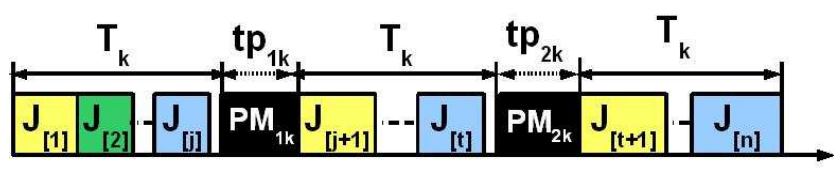

Fig. 1. A schedule on a single machine with periodic maintenance: $J_{[i]}$ is the number of job in $i$ th position and $P M_{h k}$ is the $h$ th operation of maintenance $(h=$ $1, \ldots, x)$ on machine $M_{k}$

Each Preventive Maintenance $P M_{h k}$ has a deterministic duration denoted by $t p_{h k}$ where $k$ is the index of machine. Let $x p_{h k}$ be the starting time of the maintenance $P M_{h k}$ on machine $M_{k}$. Compared to (Sbihi and Varnier (2008)) where the authors consider equal durations of maintenance tasks in the case of single machine scheduling, in this work the allocated times to maintenance operations can be different on each machine. Then the proposed dynamic (max, +) scheduling model has the starting times of operations and the starting times of maintenance activities as events of the system (see Figure 2).

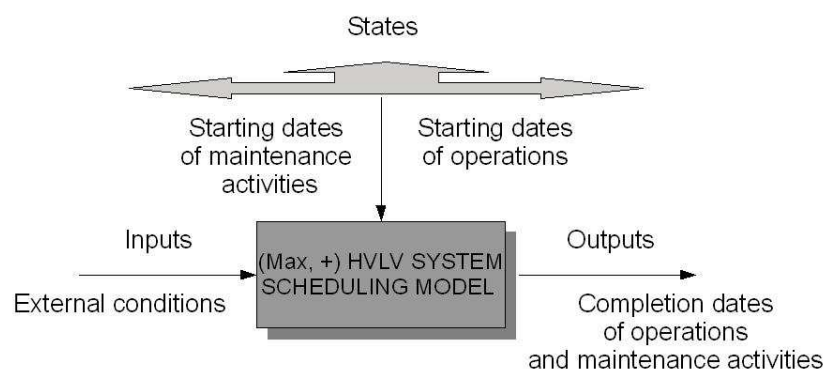

Fig. 2. HVLV systems scheduling modeling principle integrating maintenance

The proposed $(\max ,+)$ model objective is to handle simultaneously scheduling production jobs and maintenance activities. The scheduling of production jobs can be described by the same event-timing equations shown in (Nasri et al. (2011c)). Moreover, a new part is introduced into the model to represent the dynamic scheduling between maintenance activities and the scheduling between operations and maintenance activities processed on the same machines. In this case, a regular criterion (makespan) is minimized using a non-linear optimization with constraints in $(\max ,+)$ algebra. Consequently, decision variables are introduced in the model to resolve conflicts. So, the proposed model should generate all feasible schedules between operations and maintenance activities processed on the same machines.

In this paper, only periodic maintenance activities with equal periods on machines are considered. Consequently, maintenance periods are periodically fixed: maintenance is required, on each machine $M_{k}$, after a periodic time interval $T_{k}(k=1, \ldots m)$. Within this context, a (max, $+)$ HVLV model with periodic maintenance is proposed. In the latter, both the starting time of operations and maintenance tasks are considered as the events (states) of the system. Then $\forall 1 \leqslant i \leqslant n, 1 \leqslant j \leqslant n_{j}, 1 \leqslant k \leqslant m$ and $1 \leqslant h \leqslant x$, two situations can be distinguished:

If operation $j \in P$ is the first (i.e., unprecedented) operation on the job, then its processing start time $x_{i j k}$ is determined by the maximum of either:

- The starting date $t$ of the new scheduling over the new planning horizon.

- The date $u_{i}$ at which the raw material of its corresponding product $i$ is fed to the system.

- The completion of other operations $\left(j^{\prime} \neq j\right.$, and $j^{\prime} \in P$ ), for other products $i^{\prime}$ that require processing on machine $k$. This is determined by the decision variables $V_{i j k, i^{\prime} j^{\prime} k}$ that determine which operation must be processed earlier on machine $k$.

- The completion of periodic maintenance activities $P M_{h k}$ that require processing on machine $M_{k}$. This is determined by the decision variables $V_{i j k, h k}$ that generate the schedule between operation $O_{i j k}$ and the periodic maintenance $P M_{h k}$.

This situation can be formulated as follow:

$$
\begin{gathered}
x_{i j k}=\max \left(t ; u_{i} ; p_{i^{\prime} j^{\prime} k}+x_{i^{\prime} j^{\prime} k}+V_{i j k, i^{\prime} j^{\prime} k}\right. \\
\left.t p_{h k}+x p_{h k}+V_{i j k, h k}\right)
\end{gathered}
$$

Using dioid notation, the above expression may be rewritten as:

$$
\begin{aligned}
x_{i j k}= & t \oplus u_{i} \oplus p_{i^{\prime} j^{\prime} k} \otimes x_{i^{\prime} j^{\prime} k} \otimes V_{i j k, i^{\prime} j^{\prime} k} \\
& \oplus t p_{h k} \otimes x p_{h k} \otimes V_{i j k, h k}
\end{aligned}
$$

If operation $j \in P$ is not the starting operation (i.e., has predecessors) on the job, then its processing start time $x_{i j k}$ is determined by the maximum of either:

- The completion time of its direct predecessor, say $n \in P$, being processed on its correspondent machine, say $l \in M$.

- The completion of other operations $\left(j^{\prime} \neq j\right.$, and $j^{\prime} \in P$ ), for other products $i^{\prime}$ that require processing on machine $k$. This is determined by the decision variables $V_{i j k, i^{\prime} j^{\prime} k}$ that determine which operation must be processed earlier on machine $k$.

- The completion of periodic maintenance activities $P M_{h k}$ that require processing on machine $M_{k}$. This is determined by the decision variables $V_{i j k, h k}$ that 
generate the schedule between operation $O_{i j k}$ and the periodic maintenance $P M_{h k}$.

This situation can be formulated as follow:

$$
\begin{gathered}
x_{i j k}=\max \left(p_{i n l}+x_{i n l} ; p_{i^{\prime} j^{\prime} k}+x_{i^{\prime} j^{\prime} k}+V_{i j k, i^{\prime} j^{\prime} k}\right. \\
\left.t p_{h k}+x p_{h k}+V_{i j k, h k}\right)
\end{gathered}
$$

Using dioid notation, the above expression may be rewritten as:

$$
\begin{aligned}
x_{i j k}= & p_{i n l} \otimes x_{i n l} \oplus p_{i^{\prime} j^{\prime} k} \otimes x_{i^{\prime} j^{\prime} k} \otimes V_{i j k, i^{\prime} j^{\prime} k} \\
& \oplus t p_{h k} \otimes x p_{h k} \otimes V_{i j k, h k}
\end{aligned}
$$

In order to schedule the periodic maintenance activities and operations and maintenance tasks between each other that need processing on the same machine $M_{k}$, the following event-timing equation is added to the model:

$$
\begin{aligned}
& x p_{h k}=\max ( p_{i j k}+x_{i j k}+V_{h k, i j k} ; \\
& t p_{z k}+x p_{z k}+T_{k}+V_{h k, z k}
\end{aligned}
$$

- In (equation 8), the term " $p_{i j k}+x_{i j k}+V_{h k, i j k}$ " represents the sequencing between operation $O_{i j k}$ and periodic maintenance $P M_{h k}$ via the decision variable $V_{h k, i j k}$

- The term " $t p_{z k}+x p_{z k}+T_{k}+V_{h k, z k}$ " represents the scheduling of maintenance operations that need processing on machine $M_{k}$ between each other using the decision variable $V_{h k, z k}$, so that the time interval between two consecutive maintenance tasks is equal to the period $T_{k}$.

Using $(\max ,+)$ algebra notation, equation 8 becomes:

$$
\begin{aligned}
x p_{h k}= & p_{i j k} \otimes x_{i j k} \otimes V_{h k, i j k} \\
& \oplus t p_{z k} \otimes x p_{z k} \otimes T_{k} \otimes V_{h k, z k}
\end{aligned}
$$

To get feasible schedules, the different control variables in the model must be bounded and satisfy the following conditions:

$$
\begin{gathered}
V_{i j k, i^{\prime} j^{\prime} k}+V_{i^{\prime} j^{\prime} k, i j k}=B \\
\max \left(V_{i j k, i^{\prime} j^{\prime} k} ; V_{i^{\prime} j^{\prime} k, i j k}\right)=0 \\
V_{i j k, h k}+V_{h k, i j k}=B \\
\max \left(V_{i j k, h k} ; V_{h k, i j k}\right)=0 \\
V_{h k, z k}+V_{z k, h k}=B \\
\max \left(V_{h k, z k} ; V_{z k, h k}\right)=0
\end{gathered}
$$

Using $(\max ,+)$ notation, the above equations become:

$$
\begin{aligned}
& V_{i j k, i^{\prime} j^{\prime} k} \otimes V_{i^{\prime} j^{\prime} k, i j k}=B \\
& V_{i j k, i^{\prime} j^{\prime} k} \oplus V_{i^{\prime} j^{\prime} k, i j k}=0
\end{aligned}
$$

$$
V_{i j k, h k} \otimes V_{h k, i j k}=B
$$

$$
V_{i j k, h k} \oplus V_{h k, i j k}=0
$$

$$
V_{h k, z k} \otimes V_{z k, h k}=B
$$

$$
V_{h k, z k} \oplus V_{z k, h k}=0
$$

where $B$ is chosen small enough and the different decision variables are less than or equal to zero.

The period $T_{k}$ is incorporated in the model, such that the duration between two consecutive periodic maintenance activities is equal to $T_{k}$. In addition, the first maintenance activity must start at date $T_{k}$. Consequently, the following $(\max ,+)$ relations are introduced to the model:

$$
\begin{gathered}
\max \left(-x p_{1 k},-x p_{2 k}, \ldots,-x p_{x k}\right)=-T_{k} \\
\max \left(\begin{array}{c}
x p_{1 k}+t p_{1 k} ; x p_{2 k}+t p_{2 k} ; \ldots ; \\
\left.x p_{x k}+t p_{x k}\right)=\sum_{h=1}^{x} t p_{h k}+T_{k} * x
\end{array}\right.
\end{gathered}
$$

Using $(\max ,+)$ algebra notation, we have:

$$
\begin{gathered}
\bigoplus_{h=1}^{x}-x p_{h k}=-T_{k} \\
\bigoplus_{h=1}^{x}\left(x p_{h k} \otimes t p_{h k}\right)= \\
\bigotimes_{h=1}^{x} t p_{h k} \underbrace{\otimes T_{k} \otimes T_{k} \otimes . . \otimes T_{k}}_{x \text { times }}
\end{gathered}
$$

where $1 \leqslant k \leqslant m, x$ is the number of periodic maintenance activities on machine $M_{k}$.

\section{ILLUSTRATIVE EXAMPLE}

\subsection{System representation}

For the sake of simplicity and without loss of generality, the application of the $(\max ,+)$ model proposed in the Section 3 is explored below with an example of $(6 \times 6)$ JobShop system (6 products and 6 machines) (see Figure 3 ).

This example is taken from a real factory environment (Wang and Tang (2011)). It describes a very well HVLV system due to the wide variety of products (six kinds of products) and the processing times which are relatively long. Data about routing and processing times for the 6 products are presented in Table 1.

In this example, the operations of six jobs are scheduled on six machines (Table 1 ) and we have 36 state variables $x_{i j k}$ $(i=1: 6, j=1: 36$ and $k=1: 6)$. In this section, PM is considered. Then, a non-linear optimization problem with constraints is applied to minimize the makespan. 


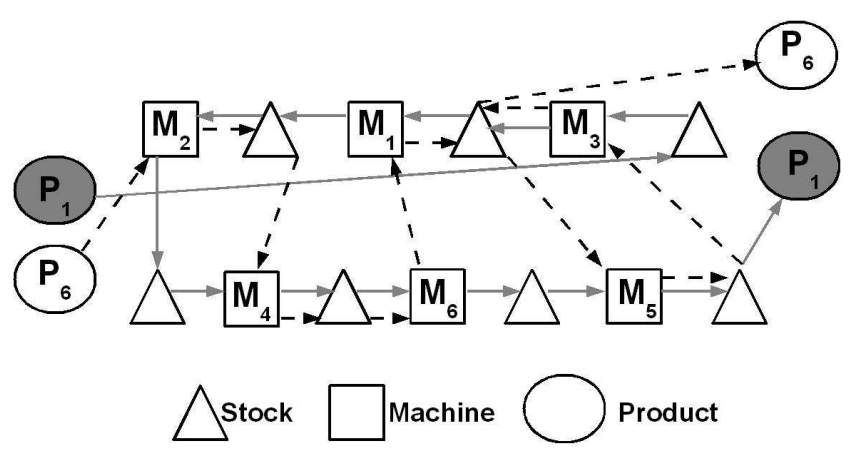

Fig. 3. Job-shop HVLV system

Table 1. Production data

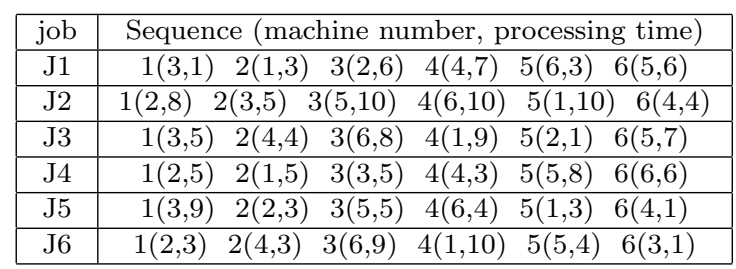

We consider here two tasks of periodic maintenance on machine $M_{1}\left(P M_{11}\right.$ and $\left.P M_{21}\right)$, two operations of periodic maintenance on machine $M_{3}\left(P M_{13}\right.$ and $\left.P M_{23}\right)$, two operations of periodic maintenance on machine $M_{4}$ $\left(P M_{14}\right.$ and $\left.P M_{24}\right)$, three tasks of periodic maintenance on machine $M_{2}\left(P M_{12}, P M_{22}\right.$ and $\left.P M_{32}\right)$, three operations of periodic maintenance on machine $M_{5}\left(P M_{15}, P M_{25}\right.$ and $\left.P M_{35}\right)$ and three periodic activities of maintenance on machine $M_{6}\left(P M_{16}, P M_{26}\right.$ and $\left.P M_{36}\right)$. Tables 2 and 3 show the PM data:

Table 2. Duration of maintenance tasks data

\begin{tabular}{|c|c|}
\hline Maintenance durations & Values \\
\hline$t p_{11}, t p_{23}, t p_{36}$ & 4 \\
\hline$t p_{21}, t p_{14}, t p_{16}, t p_{26}$ & 3 \\
\hline$t p_{12}, t p_{15}, t p_{25}$ & 2 \\
\hline$t p_{22}$ & 5 \\
\hline$t p_{32}$ & 7 \\
\hline$t p_{13}$ & 1 \\
\hline$t p_{24}, t p_{35}$ & 6 \\
\hline
\end{tabular}

Table 3. Periods of maintenance on machines

\begin{tabular}{|c|c|}
\hline Periods & Values \\
\hline$T_{1}$ & 8 \\
\hline$T_{2}$ & 9 \\
\hline$T_{3}$ & 10 \\
\hline$T_{4}$ & 12 \\
\hline$T_{5}$ & 17 \\
\hline$T_{6}$ & 20 \\
\hline
\end{tabular}

\subsection{Non-linear optimization methodology}

In this section, the proposed model is used to resolve the scheduling of the HVLV system with PM. Then, a nonlinear optimization problem with constraints is applied. It deals with the minimization of the makespan.

The objective of this section is to minimize the makespan using a non-linear optimization with constraints in (max, $+)$ algebra while preventive maintenance activities are considered (see Figure 4).

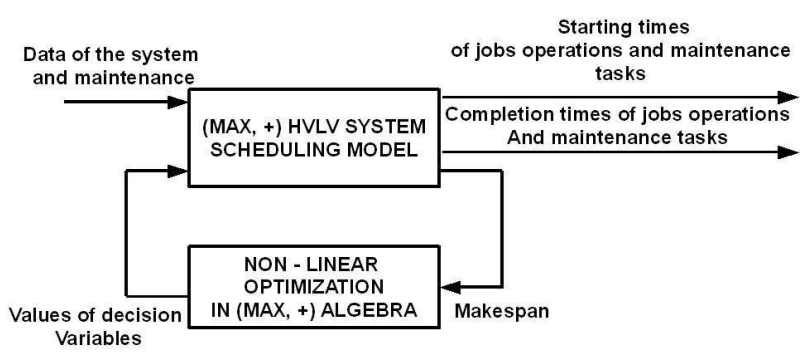

Fig. 4. Full scheme of dynamic makespan optimization

Let us now define the makespan criterion of the HVLV system as follow:

$$
C_{\max }=\max \left(C_{i}\right)=\max \left(x_{i w k}+p_{i w k}\right)
$$

where $C_{i}$ is the completion time of product $i$ and $w$ is the last operation of product $i$.

Using the $(\max ,+)$ notation, it obtains :

$$
C_{\text {max }}=\bigoplus_{i=1}^{n} C_{i}=\bigoplus_{i=1}^{n}\left(x_{i w k} \otimes p_{i w k}\right)
$$

where $x_{i w k}$ is the starting time of the last operation $w$ of product $i$ on machine $k$ and $p_{i w k}$ is its corresponding processing time. Then, the non-linear optimization scheduling problem into $(\max ,+)$ algebra is defined as:

$$
C_{\max }^{*}=\min C_{\max }=\min \left(\max \left(x_{i w k}+p_{i w k}\right)\right)
$$

Subject to the non-linear constraints (equations): (4), (6), (8), (10)-(15), (22) and (23). $B$ is chosen small enough.

Applying the non-linear optimization problem to the example of $(6 \times 6)$ Job-Shop HVLV system shown in Section 4.1 with $t=u_{i}=0$ for $i=1: 6$. Then, the obtained optimal value of the makespan $C_{\max }^{*}=\max \left(x_{165}+\right.$ $p_{165} ; x_{264}+p_{264} ; x_{365}+p_{365} ; x_{466}+p_{466} ; x_{564}+p_{564} ; x_{663}+$ $\left.p_{663}\right)=67$ time units. The corresponding schedules on the different machines based on the proposed $(\max ,+)$ model are shown in Figure 5 that shows the order of each job $J i$ on each machine $M_{k}$.

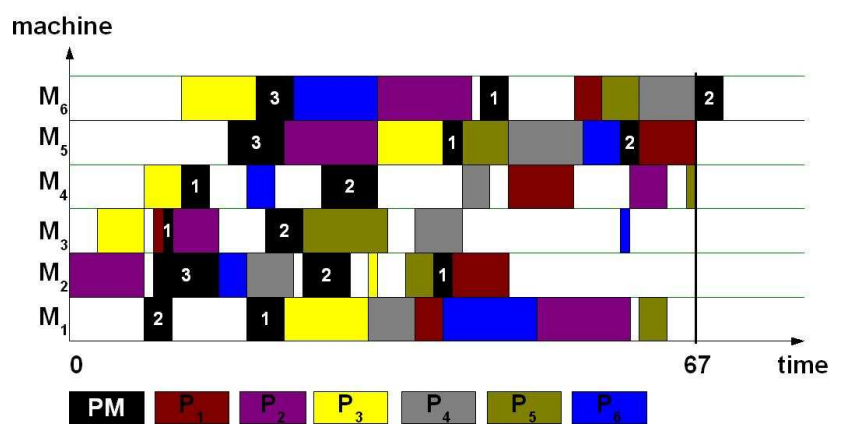

Fig. 5. Operations scheduling on the machines with PM

The completion times $C_{i}$ of the different products $i=1: 6$ are presented in Table 4.

The proposed model associated to a non-linear optimization algorithm in $(\max ,+)$ algebra leads to an optimal 
Table 4. Completion times of jobs

\begin{tabular}{|c|c|c|c|c|c|c|}
\hline Jobs & $\mathrm{J} 1$ & $\mathrm{~J} 2$ & $\mathrm{~J} 3$ & $\mathrm{~J} 4$ & $\mathrm{~J} 5$ & $\mathrm{~J} 6$ \\
\hline$C_{i}$ & 67 & 64 & 40 & 67 & 67 & 60 \\
\hline
\end{tabular}

value of the makespan $C_{\max }^{*}=67$ time units. A little comparison between this result and the value of the optimal makespan in the static case (without PM) (Nasri et al. (2011c)), shows that the incorporation of PM in the model increases the minimal value of the makespan (Figure 6).

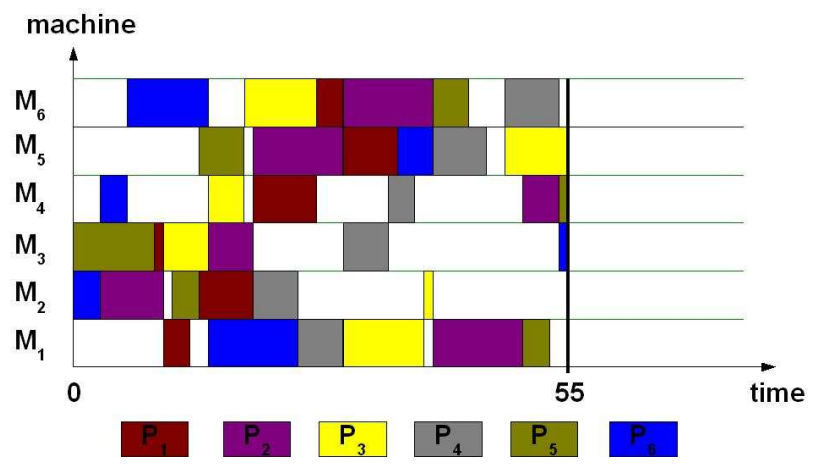

Fig. 6. Operations scheduling on the machines without PM

Figure 5 shows that the proposed $(\max ,+)$ model is efficient and valid. We can look in the diagram of gantt that the periods between maintenance tasks are respected on each machine. Also, the jobs and the maintenances are scheduled simultaneously and the maintenance activities are scheduled between each other, so that the makespan is minimal.

\section{CONCLUSION}

The objective of this work is to build a $(\max ,+)$ algebraic model for scheduling, optimization, and control of HVLV systems while periodic preventive maintenance is considered. Linear $(\max ,+)$ models cannot represent a scheduling problem for a not-decision-free systems. The non-linearity into dioid algebra is needed for HVLV systems scheduling. The proposed model incorporates decision (control) variables to resolve conflicts between concurrent operations on the same machine and between maintenance tasks. The jobs and the maintenance operations are scheduled simultaneously. Moreover, the maintenance tasks are scheduled between them, such that a regular criterion is optimized. A non-linear optimization problem with constraints is then solved into $(\max ,+)$ algebra to minimize makespan. The simulation results show that the proposed model can be an interesting tool for the control and optimization of HVLV systems integrating time based maintenance.

In real-world applications for HVLV systems, various uncertainty aspects of the system will perturb its behavior (processing times, set-up times, etc). In this context, next research work will be done to improve the proposed model to make it robust in presence of perturbations. In addition, we will try to deal with the case of flexible periodic maintenance (time intervals between two consecutive maintenances are not equals but they are known in advance). Moreover, the minimization of the total tardiness with PM in order to satisfy the Just-In-Time (JIT) production will be taken into account.

\section{REFERENCES}

Baccelli, F., Cohen, G., Olsder, G., and Quadrat, J. (1992). Synchronization and linearity. Wiley New York.

Dolgui, A., Gordon, V., and Strusevich, V. (2012). Single machine scheduling with precedence constraints and positionally dependent processing times. Computers $\&$ Operations Research, 39, 1218-1224.

Houssin, L., Lahaye, S., and Boimond, J.L. (2007). Just in time control of constrained (max,+)-linear systems. Discrete Event Dynamic Systems, 17(2), 159-178.

Huang, H. and Irani, S. (2003). An enhanced systematic layout planning process for high-variety low-volume (HVLV) manufacturing facilities. In The 17th International Conference on Production Research.

Kusiak, A. and Ahn, J. (1992). Intelligent scheduling of automated machining systems. Computer Integrated Manufacturing Systems, 5, Issue 1, 3-14.

Nasri, I., Boukezzoula, R., and Habchi, G. (2011a). A propos de la modélisation et le pilotage des systèmes manufacturiers de type HVLV. In 12ème Congrès Annuel de la Société Franaise de Recherche Opérationnelle et aide à la Décision (ROADEF'11), Saint-Etienne, France, 2-4 March, N383, Page II-665.

Nasri, I., Habchi, G., and Boukezzoula, R. (2011b). Max-plus-linear model-based predictive control for constrained HVLV manufacturing systems. In 16th IEEE International Conference on Emergency Technologies and Factory Automation (ETFA'11), Toulouze, France, 5-9 Septembre 2011. Pages 1-4.

Nasri, I., Habchi, G., and Boukezzoula, R. (2011c). Scheduling and control modeling of HVLV systems using max-plus algebra. In 5th International Workshop on Verification and Evaluation of Computer and Communication Systems (VECoS'11), Tunis, Tunisia, 15-16 Septembre 2011. Pages 62-70.

Sbihi, M. and Varnier, C. (2008). Single-machine scheduling with periodic and flexible periodic maintenance to minimize maximum tardiness. Computers and Industrial Engineering, 55, 830-840.

Tamani, K., Boukezzoula, R., and Habchi, G. (2009). Intelligent distributed and supervised flow control methodology for production systems. Engineering Applications of Artificial Intelligence, 22, 1104-1116.

Tamani, K., Boukezzoula, R., and Habchi, G. (2011a). Application of a continuous supervisory fuzzy control on a discrete scheduling of manufacturing systems. Engineering Applications of Artificial Intelligence (EAAI), 24, N7, 1162-1173.

Tamani, K., Boukezzoula, R., and Habchi, G. (2011b). Multi-objective supervisory flow control based on fuzzy interval arithmetic: application for scheduling of manufacturing systems. Simulation Practices Modeling and Theory Journal (SIMPAT), 19, N5, 13711383.

Wang, L. and Tang, D. (2011). An improved adaptive genetic algorithm based on hormone modulation mechanism for job-shop scheduling problem. Expert Systems with Applications, 38, 7243-7250.

Zribi, N., Kamel, A., and Borne, P. (2008). Minimizing the makespan for the mpm job-shop with availability constraints. International Journal of Production Economics, 112, 151-160. 